\title{
Macular hole surgery with short-acting gas and short-duration face-down positioning
}

This article was published in the following Dove Press journal:

Clinical Ophthalmology

19 July 2012

Number of times this article has been viewed

\author{
Tina Xirou' \\ Panagiotis G Theodossiadis ${ }^{2}$ \\ Michael Apostolopoulos ${ }^{3}$ \\ Stamatina A Kabanarou' \\ Elias Feretis' \\ loannis D Ladas ${ }^{3}$ \\ Chrysanthi Koutsandrea ${ }^{3}$ \\ 'Vitreoretinal Unit, Red Cross \\ Hospital, ${ }^{2} \mathrm{~B}$ Department of \\ Ophthalmology, University of \\ Athens, Greece; ${ }^{3} \mathrm{~A}$ Department \\ of Ophthalmology, University \\ of Athens, Greece
}

Purpose: To report on the outcomes of vitrectomy and sulfur hexafluoride $\left(\mathrm{SF}_{6}\right)$ gas tamponade for idiopathic macular holes with 2 days of face-down positioning.

Patients and methods: This was a prospective, nonrandomized, observational sequential case-series study on 23 consecutive patients receiving macular hole surgery using $20 \% \mathrm{SF}_{6}$ and advised to stay in a face-down position for 2 days postoperatively ( $\mathrm{SF}_{6}$ group). These patients were compared to 23 consecutive patients who had previously undergone macular hole surgery, had received $14 \% \mathrm{C}_{3} \mathrm{~F}_{8}$, and were advised to maintain a face-down position for 2 days $\left(\mathrm{C}_{3} \mathrm{~F}_{8}\right.$ group). Patients in both groups underwent vitrectomy, internal limiting membrane peeling, and fluid gas exchange using either $\mathrm{SF}_{6}$ or $\mathrm{C}_{3} \mathrm{~F}_{8}$. Preoperative and postoperative data included best corrected visual acuity recorded in LogMAR units, slit-lamp biomicroscopy, and optical coherence tomography.

Results: At a 6-month follow-up, macular hole closure was noted in 23/23 eyes (100\%) and in $22 / 23$ eyes (96\%) in the $\mathrm{SF}_{6}$ and $\mathrm{C}_{3} \mathrm{~F}_{8}$ groups, respectively. The improvement in visual acuity (measured through Snellen acuity lines both preoperatively until 6 months postoperatively) was $4.08 \pm 2.31$ (95\% confidence interval [CI]: 3.08-5.08) for the $\mathrm{SF}_{6}$ group and $2.87 \pm 2.30(95 \%$ CI: $1.87-3.86)$ for the $\mathrm{C}_{3} \mathrm{~F}_{8}$ group; this difference was not statistically significant $(P=0.06)$.

Conclusion: Vitrectomy with internal limiting membrane peeling and a short-acting gas tamponade using $\mathrm{SF}_{6}$ with posture limitation for 2 days may give a high success rate in macular hole surgery.

Keywords: idiopathic macular holes, $\mathrm{SF}_{6}$ gas tamponade, $\mathrm{C}_{3} \mathrm{~F}_{8}$ gas tamponade

\section{Introduction}

Macular hole surgery (MHS) is a well-established method for the treatment of idiopathic macular holes. ${ }^{1}$ The initial protocol for MHS was a pars plana vitrectomy, posterior vitreous removal, filling of the vitreous cavity with a gas bubble, and postoperative face-down positioning for 1 week. ${ }^{2}$ To maximize the chance of a successful procedure, a long-lasting gas tamponade and maintenance of face-down positioning for several days is standard practice in MHS. ${ }^{3}$ However, this practice is not easily tolerated by elderly patients.

The current study aims to examine the surgical outcomes of macular hole surgery with the use of a short-acting gas and limited-duration face-down positioning.

\section{Patients and methods}

This study was approved by the Ethical Committee of the District General Hospital Geniko Kratiko in Athens. It was a prospective, nonrandomized, observational 
sequential case-series study of patients undergoing vitrectomy with internal limiting membrane (ILM) peel, gas tamponade, and two days' postoperative face-down positioning. The procedures were performed from March 2008 through January 2011 by one surgeon (TX). Twenty-three consecutive patients underwent MHS using a gas bubble composed of $20 \% \mathrm{SF}_{6}$, and were advised to remain in a face-down position for 2 days postoperatively (these patients are referred to as the $\mathrm{SF}_{6}$ group). These patients were compared to 23 consecutive patients who had previously undergone MHS with a gas bubble composed of $14 \% \mathrm{C}_{3} \mathrm{~F}_{8}$, and were advised to remain in the same face-down position for 2 days postoperatively (the $\mathrm{C}_{3} \mathrm{~F}_{8}$ group).

All patients in the study had idiopathic macular holes from stages 2 to 4 as determined by biomicroscopy and optical coherence tomography (OCT) imaging. ${ }^{4,5}$ Exclusion criteria were macular holes due to trauma and high myopia. Preoperative data were collected, including age, sex, bestcorrected visual acuity (BCVA, recorded in LogMAR units), slit-lamp biomicroscopy and OCT macular thickness analysis (Stratus 3; Carl Zeiss Meditec AG, Jena, Germany). The minimum diameter of the macular hole, the base diameter of the macular hole, and hole height were measured. Macular holes were graded according to OCT in stages 2, 3, and 4 (Tables 1 and 2). ${ }^{5}$

All patients underwent a three-port 20-gauge pars plana vitrectomy, followed by surgical induction of posterior vitreous detachment (PVD) and extensive removal of peripheral vitreous up to the vitreous base. All patients received fluid-air exchange to facilitate ILM staining with

Table I Sex and age of patients, mean distance between macular hole edges, mean diameter of the hole base, mean hole height, and the macular hole stage documented for the $\mathrm{C}_{3} \mathrm{~F}_{8}$ group, including $95 \% \mathrm{Cl}$ and $P$ values

\begin{tabular}{ll}
\hline $\mathbf{C}_{3} \mathbf{F}_{\mathbf{8}}(\mathbf{n}=\mathbf{2 3})$ & \\
Gender (male) & $10(43 \%)$ \\
Age (years) & Mean: $70.6 \pm 6.2$ \\
& $95 \%$ Cl: 67.9 to 73.3 \\
Average edge $(\mu \mathrm{m})$ & Mean: $426.2 \pm 155.3$ \\
& $95 \%$ Cl: 359.0 to 493.3 \\
Average base $(\mu \mathrm{m})$ & Mean: $833.2 \pm 326.2$ \\
& $95 \%$ Cl: 692.1 to 974.2 \\
Average height $(\mu \mathrm{m})$ & Mean: $377.5 \pm 77.9$ \\
& $95 \% \mathrm{Cl}: 343.8$ to 411.2 \\
Stage & Stage $2:(n=7,30.4 \%)$ \\
& Stage $3:(n=12,52.2 \%)$ \\
& Stage $4:(n=4,17.4 \%)$ \\
\hline
\end{tabular}

Abbreviation: $\mathrm{Cl}$, confidence interval.
Table 2 Sex and age of patients, mean distance between macular hole edges, mean diameter of the hole base, mean of hole height, and macular hole stage documented for the $\mathrm{SF}_{6}$ group, including $95 \% \mathrm{Cl}$ and $\mathrm{P}$ values

\begin{tabular}{ll}
\hline $\mathbf{S F}_{\mathbf{6}}(\mathbf{n}=\mathbf{2 3})$ & \\
Gender $($ male $)$ & 12 (52\%) \\
Age (years) & Mean: $69.5 \pm 7.4$ \\
& $95 \% \mathrm{Cl}: 66.3$ to 72.7 \\
Average edge $(\mu \mathrm{m})$ & Mean: $385.0 \pm 165.5$ \\
& $95 \% \mathrm{Cl}: 313.5$ to 456.6 \\
Average base $(\mu \mathrm{m})$ & Mean: $795.1 \pm 289.1$ \\
& $95 \% \mathrm{Cl}: 670.1$ to 920.2 \\
Average height $(\mu \mathrm{m})$ & Mean: $385.6 \pm 84.2$ \\
& $95 \% \mathrm{Cl}: 349.1$ to 422.0 \\
Stage & Stage $2:(\mathrm{n}=19,82.6 \%)$ \\
& Stage $3:(\mathrm{n}=16,17.4 \%)$ \\
& Stage $4:(\mathrm{n}=0,0 \%)$ \\
\hline
\end{tabular}

Abbreviation: $\mathrm{Cl}$, confidence interval.

brilliant peel. One drop of the dye was applied over the surface of the macula, painting the area between the vascular arcades. Using passive aspiration, the dye could be easily removed after 10 to 15 seconds without reducing the quality of visualization. After the dye was removed and air-fluid exchange was performed with the help of a micropick needle, the first flap was made temporal to the fovea and the ILM was peeled with Eckardt's forceps to one disk diameter away from the foveal center. At the end of surgery, careful inspection of the retinal periphery was made to exclude peripheral breaks, and the eye was flushed with $50 \mathrm{~mL}$ of either $14 \% \mathrm{C}_{3} \mathrm{~F}_{8}$ or $20 \% \mathrm{SF}_{6}$. The patients were placed in a face-down position overnight, and were advised to remain face-down for one more day at home. All patients were examined in the outpatient clinic 10 days postoperatively, where further clinical evaluation through biomicroscopy and measurements of BCVA and OCT macular thickness were performed. Further follow-up appointments were made at 1 , 3 , and 6 months after surgery.

\section{Results}

There was a 13:10 ratio of females to males in the $\mathrm{C}_{3} \mathrm{~F}_{8}$ group in contrast to a 11:12 ratio in the $\mathrm{SF}_{6}$ group $(P=0.55$, chi-square test). The mean ages of the two groups were 70.6 years and 69.5 years, respectively $(P=0.56$, $t$-test). The $\mathrm{C}_{3} \mathrm{~F}_{8}$ group had fewer stage 2 and 3 macular holes than the $\mathrm{SF}_{6}$ group $(P=0.0004$, chi-squared test). Stage 4 macular holes were presented only on the $\mathrm{C}_{3} \mathrm{~F}_{8}$ group (17.4\%). Mean distance between macular hole edges and mean diameter of the hole base were 
greater in the $\mathrm{C}_{3} \mathrm{~F}_{8}$ group, though this difference was not statistically significant $(P=0.39$ and $P=0.68$, respectively, both analyses $t$-tests). Mean average hole height was less in the $\mathrm{C}_{3} \mathrm{~F}_{8}$ group, though this was not a statistically significant difference ( $P=0.73$, $t$-test) (Tables 1 and 2$)$.

The preoperative mean BCVA (LogMAR) distributions of the $\mathrm{C}_{3} \mathrm{~F}_{8}$ and $\mathrm{SF}_{6}$ groups differed significantly $(P<0.0001$, two-sample $t$-test), and were $0.90 \pm 0.10$ (20/158 Snellen acuity; 95\% confidence interval [CI]: 0.86-0.95) and $0.67 \pm 0.20$ (20/93 Snellen acuity; 95\% CI: 0.58-0.76), respectively. Macular hole closure was achieved in all 23 eyes ( $100 \%$ anatomical success rate) in the $\mathrm{SF}_{6}$ group and in 22 eyes $(96 \%)$ in the $\mathrm{C}_{3} \mathrm{~F}_{8}$ group.

Anatomical closure was established earlier in the $\mathrm{SF}_{6}$ group, as the gas bubble was absorbed sooner (median 14 days postoperatively) than in the $\mathrm{C}_{3} \mathrm{~F}_{8}$ group (median 45 days). Macular hole closure was confirmed by day 10 in the $\mathrm{SF}_{6}$ group, when patients were given an OCT examination, and their fovea was uncovered from gas. Patients from the $\mathrm{C}_{3} \mathrm{~F}_{8}$ group needed a much longer period before being uncovered.

Postoperative mean BCVA (LogMAR) 6 months after surgery was significantly improved in both groups. For the $\mathrm{C}_{3} \mathrm{~F}_{8}$ group the mean was $0.62 \pm 0.23$ (20/83 Snellen acuity; 95\% CI: 0.53-0.72) with 0.28 LogMAR improvement $(P<0.0001)$. For the $\mathrm{SF}_{6}$ group the mean was $0.29 \pm 0.12$ (20/38 Snellen acuity; 95\% CI: 0.24-0.34) with 0.38 LogMAR improvement $(P<0.0001)$. The LogMAR improvement for the $\mathrm{SF}_{6}$ group was significantly greater than for the $\mathrm{C}_{3} \mathrm{~F}_{8}$ group $(P<0.0001)$. Since the distributions of $\operatorname{LogMAR}$ postoperative BCVAs follow a normal distribution for both gases and for both stages 2 and 3, changes between stages were examined using $t$-tests. The results for stage 2 differed significantly, with a mean BCVA value of $0.57 \pm 0.27$ (95\% CI: $0.33-0.82)$ for the $\mathrm{C}_{3} \mathrm{~F}_{8}$ group and $0.26 \pm 0.12(95 \%$ CI: $0.21-0.32)$ for the $\mathrm{SF}_{6}$ group $(P=0.023)$. The results for stage 3 were also statistically significant and showed a mean BCVA of $0.61 \pm 0.22$ (95\% CI: $0.47-0.75)$ for the $\mathrm{C}_{3} \mathrm{~F}_{8}$ group and $0.39 \pm 0.09$ (95\% CI: $\left.0.25-0.54\right)$ for the $\mathrm{SF}_{6}$ group $(P=0.014)$ (Table 3$)$.

The improvement in Snellen acuity 6 months postoperatively was $2.87 \pm 2.30$ (95\% CI: $1.87-3.86)$ for the $\mathrm{C}_{3} \mathrm{~F}_{8}$ group and $4.08 \pm 2.31$ (95\% CI: $3.08-5.08)$ for the $\mathrm{SF}_{6}$ group, although this difference was not statistically significant $(P=0.06$, Wilcoxon rank sum test). Fifty-two percent of eyes (12 eyes) in the $\mathrm{C}_{3} \mathrm{~F}_{8}$ group experienced a halving of the visual angle (approximately a three Snellen line improvement), as did $78 \%$ of eyes (18 eyes) in the $\mathrm{SF}_{6}$ group.
To evaluate the predictive factors for the observed visual outcomes, the following variables were included in logistic regression models for pre- or postoperative risk factors for BCVA improvement for more than three lines at 3 months: age, gas type used, sex, preoperative BCVA, and stage of macular hole. Sex, age, and preoperative macular hole stage were all found to have nonstatistically significant roles in the designed model; only preoperative BCVA and the type of gas used were found to be significant, as was expected. The gas was found to have borderline significance $(P=0.046$, chi-square test), while the preoperative BCVA was found to be significantly associated with postoperative visual acuity improvement $(P=0.018)$.

There were no observed incidences of endophthalmitis or retinal detachment. Elevation of intraocular pressure $(>30 \mathrm{mmHg}$ ) was noted on the first postoperative day in three patients in each group, which was controlled with topical therapy. Intraoperative retinal breaks were detected in two patients in the $\mathrm{C}_{3} \mathrm{~F}_{8}$ group, and these were treated with cryotherapy.

\section{Discussion}

Idiopathic macular hole is a retinal disease mainly affecting elderly patients. No effective treatment was available until the early 1990s when Kelly and Wendel described their technique, which included vitrectomy with induced posterior vitreous detachment, gas tamponade, and strict face-down positioning for 1 week. ${ }^{2}$ Since then, many alternatives to this basic protocol have been proposed and have allowed the successful closure rate to increase to more than $90 \% .{ }^{6}$ Nevertheless, the necessity of ILM staining/peeling, duration of gas tamponade, and postoperative head positioning are still a matter of controversy.

Gas tamponade plays an important role in hole closure during macular hole surgery, as the gas provides a scaffold for glial proliferation, and its surface tension may exclude vitreous fluid from the subretinal space. Flotation forces do not seem to play a significant role in macular hole closure. ${ }^{7,8}$ To maximize the effects of the gas, a long-acting gas and a long duration of face-down positioning have both been used. Patients may become distressed and avoid surgery if advised to maintain a face-down position for a week, or if they are unable to resume normal activities or travel for a few weeks postoperatively. Over the years, vitreoretinal surgeons have tried to increase patients' postoperative comfort by decreasing the duration of gas tamponade or the length of time in which patients remain face down, but these changes have produced unconvincing results. ${ }^{9,10}$ 
Table 3 Patients' mean pre- and postoperative (6 month) best-corrected visual acuity (LogMAR) were plotted according to the gases used and macular hole stage

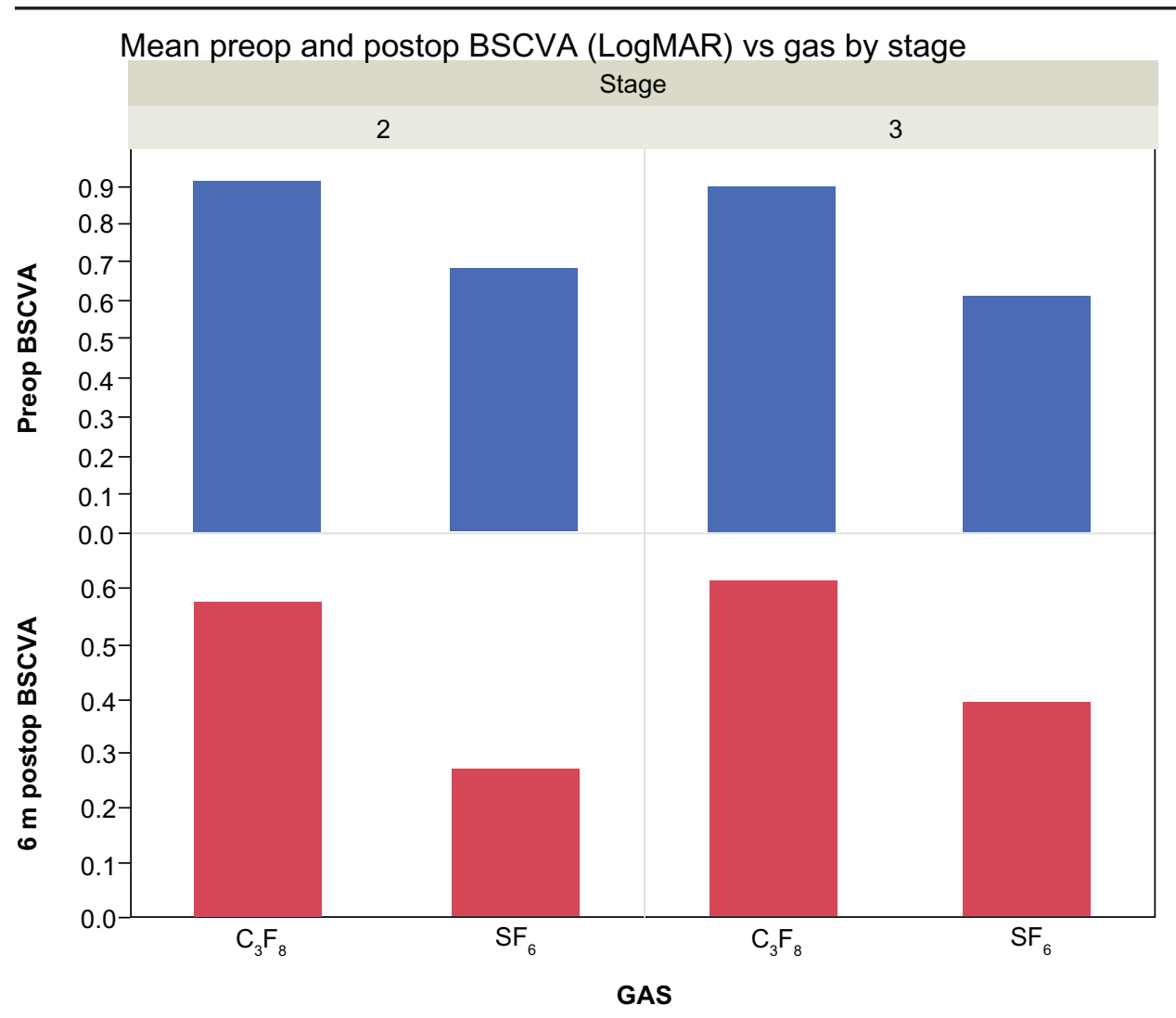

Notes: For the $\mathrm{C}_{3} \mathrm{~F}_{8}$ group, the mean value was $0.62 \pm 0.23$ ( $\left.95 \% \mathrm{Cl}: 0.53-0.72\right)$ with 0.28 LogMAR improvement $(P<0.000 \mathrm{I})$. For the $\mathrm{SF}_{6}$ group, the mean value was $0.29 \pm 0.12$ (95\% Cl: $0.24-0.34)$ with 0.38 LogMAR improvement $(P<0.000 I)$. The LogMAR improvement for the $\mathrm{SF}_{6}$ group was significantly better than that of the $\mathrm{C}_{3} \mathrm{~F}_{8}$ group $(P<0.000 \mathrm{I})$. Furthermore, for macular holes stage 2 , best-corrected visual acuity was statistically significant improved $\left(0.57 \pm 0.27\right.$ [ $95 \% \mathrm{Cl}$ : $0.33-0.82$ ] for the $\mathrm{C}_{3} \mathrm{~F}_{8}$ group and $0.26 \pm 0.12$ [95\% Cl: $0.21-0.32]$ for the $\mathrm{SF}_{6}$ group $[P=0.023]$ ). For patients with stage 3 macular holes, best-corrected visual acuity also significantly improved $(0.6 \mathrm{I} \pm 0.22$ [95\% Cl: $0.47-0.75]$ for the $\mathrm{C}_{3} \mathrm{~F}_{8}$ group and $0.39 \pm 0.09$ [95\% Cl: $\left.0.25-0.54\right]$ for the $\mathrm{SF}_{6}$ group $\left.[P=0.014]\right)$.

Abbreviation: $\mathrm{Cl}$, confidence interval.

Although there is no evidence that anatomic success is better in patients undergoing a longer period of tamponade, $\mathrm{C}_{3} \mathrm{~F}_{8}$ remains the gas of choice for two thirds of vitreoretinal surgeons. ${ }^{11}$ The option of using a gas with a shorter duration has been attractive to some researchers. Kim et al used $\mathrm{SF}_{6}$ or $\mathrm{C}_{3} \mathrm{~F}_{8}$ and found similar anatomical results with both gasses, reporting a $90 \%$ macular hole closure rate for the $\mathrm{SF}_{6}$ group and $91 \%$ for the $\mathrm{C}_{3} \mathrm{~F}_{8}$ group (all patients maintained face-down positioning for 1 week postoperatively). ${ }^{11}$ Eckardt et al suggested that air could be an alternative tamponade in macular hole surgery, and found that $54 \%$ of macular holes can be closed within 24 hours postoperatively and $75.7 \%$ within 48 hours postoperatively; to increase the success rate, some patients had to be operated on more than once. ${ }^{9}$ These results were confirmed by Gesser et al who reported that the initial closure rate for air as a tamponade for macular hole surgery was only $79 \%$, approximately $10 \%$ lower than that reported in other studies employing long-acting gas tamponades. ${ }^{12}$

Other surgeons have proposed that long-lasting gas be used, and face-down positioning be avoided. ${ }^{7,8,13}$ The questions of whether face-down positioning is required at all, or whether overnight positioning is sufficient, have been investigated in several studies that have resulted in success rates of $85 \%$ to $96.7 \% .^{7,8,14-17}$ The authors of these studies used a long-acting intraocular tamponade in order to minimize the face-down positioning period, or to allow no positioning requirements at all. Only one author has reported a $100 \%$ closure rate in macular hole surgery when limiting the positioning to 1 day; in this instance $\mathrm{C}_{2} \mathrm{~F}_{6}$ was used, which may last longer than $\mathrm{SF}_{6}$, and less than $\mathrm{C}_{3} \mathrm{~F}_{8}{ }^{18}$

The indication of face-down positioning may be important when a short-lasting tamponade is chosen in macular hole surgery. This becomes apparent in studies of patients who 
received $\mathrm{SF}_{6}$ without positioning, where the success rate for macular hole closure did not exceed $89 \%-90 \%$ in one operation. ${ }^{10,15}$ In contrast, Kim et al found that macular hole surgery using $\mathrm{SF}_{6}$ gas yielded similar results to those seen with $\mathrm{C}_{3} \mathrm{~F}_{8}$ gas, but all patients had to maintain maximal facedown positioning for 1 week postoperatively. ${ }^{11}$

In the present study, an effort was made to decrease patient discomfort arising from prolonged immobility, with 2 days of face-down positioning required; as previous studies have highlighted, a total lack of face-down positioning appears to be insufficient, and closure of macular holes within 3 days of ILM peeling has been reported and confirmed by optical coherence tomography imaging. ${ }^{19}$ In the present study, the use of $\mathrm{SF}_{6}$, a short-acting gas tamponade, and a short period of 2 days face-down positioning, was found to sufficiently repair macular holes in $100 \%$ of patients. $\mathrm{C}_{3} \mathrm{~F}_{8}$ was not found to be superior with regards to closure rate for stage 2 and 3 macular holes. Stage 4 holes were present only in the $\mathrm{C}_{3} \mathrm{~F}_{8}$ group, and only one patient with a stage 4 macular hole suffered a hole-closure failure. This was attributed to the hole being of long standing, but it is noted that the length of time that a macular hole has been present is not in the exclusion criteria for surgery.

It was found that the most favorable outcomes for visual recovery were associated with better initial visual acuity, as has been reported in previous studies. ${ }^{20}$ The $\mathrm{SF}_{6}$ group had a better final acuity at 6 months, reflecting the better initial visual acuity and the smaller macular holes in these patients. Both the $\mathrm{C}_{3} \mathrm{~F}_{8}$ and $\mathrm{SF}_{6}$ groups experienced a halving of the visual angle, which equates to approximately a three-line improvement on a Snellen acuity chart. Further analysis for subgroups of stages 2 and 3 in relation to the BCVA showed significantly better outcomes for patients who received $\mathrm{SF}_{6}$, as preoperative visual acuity was better in those patients. A larger number of patients and more even preoperative baseline characteristics would be desirable to enhance the power of the results of this study.

All studied patients underwent intra-operative ILM staining with brilliant peel and ILM peeling. ${ }^{21-23}$ Although ILM peeling may not be necessary for idiopathic macular holes, most studies indicate that better anatomical and functional outcomes can be achieved after peeling. ${ }^{24-26}$ Furthermore, ILM peeling may prevent reopening of the macular hole. ${ }^{27,28}$ Many surgeons prefer to use indocyanine green to stain the ILM as this assists in visualization and increases the ease of peeling, although there are concerns regarding poorer patient outcomes associated with its use. ${ }^{29,30}$
All patients included in this study were pseudophakic or had undergone cataract extraction 2 to 4 weeks prior to macular hole surgery. Combined phacovitrectomy maybe a choice for some surgeons, but this has been associated with complications such as an iris/intraocular lens capture in up to $8.6 \%$ of patients. ${ }^{13,31}$ Cataract surgery performed as a separate procedure after macular hole repair can cause complications due to intraoperative difficulties during phacoemulsification, which arise from the absence of vitreous support in a vitrectomized eye and the increased risk of zonular or posterior capsular insult. ${ }^{7,13}$ Moreover, cataract surgery following macular hole repair may lead to postoperative reopening of the macular hole in $20 \%$ of patients, especially if there are complications involving cystoid macular edema. ${ }^{32}$

The current study was somewhat limited by a lack of randomization, the short follow-up period, and lack of stage 4 macular holes. Despite this, the study showed that a short-acting intraocular gas tamponade with two days of postoperative face-down positioning allowed macular holes to be closed with a very high success rate. This method may be a good alternative for stage 1, 2, and 3 macular holes, as it allows a short duration of visual impairment and rapid postoperative rehabilitation.

\section{Disclosure}

The authors have no proprietary interest and there was no financial support for this study.

\section{References}

1. La Cour M, Friis J. Macular holes: classification, epidemiology, natural history and treatment. Acta Ophthalmol Scand. 2002;80(6):579-587.

2. Kelly NE, Wendel RT. Vitreous surgery for idiopathic macular holes Results of a pilot study. Arch Ophthalmol. 1991;109(5):654-659.

3. Dori D, Thoelen AM, Akalp F, Bernasconi PP, Messmer EP. Anatomic and functional results of vitrectomy and long-term intraocular tamponade for stage 2 macular holes. Retina. 2003;23(1):57-63.

4. Gass JD. Reappraisal of biomicroscopic classification of stages of development of a macular hole. Am J Ophthalmol. 1995;119(6): $752-759$.

5. Azzoloni C, Patelli F, Brancato R. Correlation between optical coherence tomography data and biomicroscopic interpretation of idiopathic macular hole. Am J Ophthalmol. 2001;132(3):348-355.

6. Margherio AR. Macular hole surgery in 2000. Curr Opin Ophthalmol. 2000;11(3):186-190.

7. Tornambe PE, Poliner LS, Grote K. Macular hole surgery without face-down positioning. A pilot study. Retina. 1997;17(3):179-185.

8. Tranos PG, Peter NM, Nath R, et al. Macular hole surgery without prone positioning. Eye. 2007;21(6):802-806.

9. Eckardt C, Eckert T, Eckardt U, Porkert U, Gesser C. Macular hole surgery with air tamponade and optical coherence tomography-based duration of face-down positioning. Retina. 2008;28(8):1087-1096.

10. Yagi F, Sato Y, Takagi S, Tomita G. Idiopathic macular hole vitrectomy without postoperative face-down positioning. Jpn J Ophthalmol. 2009; 53(3):215-218. 
11. Kim SS, Smiddy WE, Feuer WJ, Shi W. Outcomes of sulfur hexafluoride (SF6) versus perfluoropropane $\left(\mathrm{C}_{3} \mathrm{~F}_{8}\right)$ gas tamponade for macular hole surgery. Retina. 2008;28(10):1408-1415.

12. Gesser C, Eckert T, Eckardt U, Porkert U, Eckardt C. Macular hole surgery with air tamponade. Does air suffice for short-term tamponade? Ophthalmologie. 2010;107(11):1043-1050. German.

13. Heath G, Rahman R. Combined 23-gauge, sutureless transconjuctival vitrectomy with phacoemulsification without face down posturing for the repair of idiopathic macular holes. Eye. 2010;24(2):214-221.

14. Simcock PR, Scalia S. Phacovitrectomy without prone posture for full thickness macular holes. Br J Ophthalmol. 2001;85(11):1316-1319.

15. Carvounis PE, Kopel AC, Kuhl DP, Heffez J, Pepple K, Holz ER. 25-guage vitrectomy using sulfur hexafluoride and no prone positioning for repair of macular holes. Retina. 2008;28(9):1188-1192.

16. Madgula IM, Costen M. Functional outcome and patient preferences following combined phaco-vitrectomy for macular hole without prone posturing. Eye. 2008;22(8):1050-1053.

17. Gupta D. Face-down posturing after macular hole surgery: a review. Retina. 2009;29(4):430-443.

18. Dhawahir-Scala FE, Maino A, Saha K, Mokashi AA, McLauchlan R, Charles S. To posture or not to posture after macular hole surgery. Retina. 2008;28(1):60-65.

19. Sato H, Kawasaki R, Yamashita H. Observation of idiopathic fullthickness macular hole closure in early postoperative period as evaluated by optical coherence tomography. Am J Ophthalmol. 2003;136(1): 185-187.

20. Gupta B, Laidlaw DAH, Williamson TH, Shah SP, Wong R, Wren S. Predicting visual success in macular hole surgery. $\mathrm{Br} J$ Ophthalmol. 2009;93(11):1488-1491.

21. Balaiya S, Brar VS, Murthy RK, Chalam KV. Comparative in vitro safety analysis of dyes for chromovitrectomy: indocyanine green, brilliant blue green, bromophenol blue, and infracyanine green. Retina. 2011;31(6):1128-1136.
22. Awad D, Schrader I, Bartok M, Mohr A, Gabel D. Comparative toxicology of trypan blue, brilliant blue $\mathrm{G}$, and their combination together with polyethylene glycol on human pigment epithelial cells. Invest Ophthalmol Vis Sci. 2011;52(7):4085-4090.

23. Enaida H, Hisatomi T, Hata Y, et al. Brilliant blue G selectively stains the internal limiting membrane/brilliant blue G-assisted membrane peeling. Retina. 2006;26(6):631-636.

24. Haritoglou C, Reiniger IW, Schaumberger M, Gass CA, Priglinger SG, Kampik A. Five-year follow-up of macular hole surgery with peeling of the internal limiting membrane: update of a prospective study. Retina. 2006;26(6):618-622.

25. Christensen UC. Value of internal limiting membrane peeling in surgery for idiopathic macular hole and the correlation between function and retinal morphology. Acta Ophthalmol. 2009;87 Thesis 2:1-23.

26. Kimura T, Takahashi $\mathrm{M}$, Takagi $\mathrm{H}$, et al. Is removal of internal limiting membrane always necessary during stage 3 idiopathic macular hole surgery? Retina. 2005;25(1):54-58.

27. Kumagai K, Furukawa M, Ogino N, Uemura A, Demizu S, Larson E. Vitreous surgery with and without internal limiting membrane peeling for macular hole repair. Retina. 2004;24(5):721-727.

28. Yoshiba M, Kishi S. Pathogenesis of macular hole recurrence and its prevention by internal limiting membrane peeling. Retina. 2007;27(2): 169-173.

29. Slaughter K, Lee IL. Macular hole surgery with and without indocyanine green assistance. Eye. 2004;18(4):376-378.

30. Grisanti S, Altvater A, Peters S. Safety parameters for indocyanine green in vitreoretinal surgery. Dev Ophthalmol. 2008;42:43-68.

31. Smith M, Raman SV, Pappas G, Simcock P, Ling R, Shaw S. Phacovitrectomy for primary retinal detachment repair in presbyopes. Retina. 2007;27(4):462-467.

32. Bhatnagar P, Kaiser PK, Smith SD, Meisler DM, Lewis H, Sears JE. Reopening of previously closed macular holes after cataract extraction. Am J Ophthalmol. 2007;144(2):252-259.
Clinical Ophthalmology

\section{Publish your work in this journal}

Clinical Ophthalmology is an international, peer-reviewed journal covering all subspecialties within ophthalmology. Key topics include: Optometry; Visual science; Pharmacology and drug therapy in eye diseases; Basic Sciences; Primary and Secondary eye care; Patient Safety and Quality of Care Improvements. This journal is indexed on

\section{Dovepress}

PubMed Central and CAS, and is the official journal of The Society of Clinical Ophthalmology (SCO). The manuscript management system is completely online and includes a very quick and fair peer-review system, which is all easy to use. Visit http://www.dovepress.com/ testimonials.php to read real quotes from published authors. 